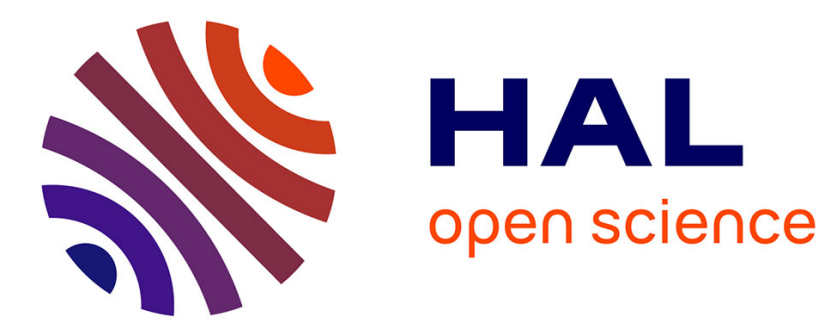

\title{
A bi-criterion approach for the airlines crew rostering problem
}

Walid El Moudani, Carlos Alberto Nunes Cosenza, Marc de Coligny, Felix Mora-Camino

\section{- To cite this version:}

Walid El Moudani, Carlos Alberto Nunes Cosenza, Marc de Coligny, Felix Mora-Camino. A bicriterion approach for the airlines crew rostering problem. EMO 2001, 1st International Conference on Evolutioary Multi-Criterion Optimization, Mar 2001, Zurich, Switzerland. pp 486-500, 10.1007/3540-44719-9_34. hal-01021705

\section{HAL Id: hal-01021705 \\ https://hal-enac.archives-ouvertes.fr/hal-01021705}

Submitted on 10 Jul 2014

HAL is a multi-disciplinary open access archive for the deposit and dissemination of scientific research documents, whether they are published or not. The documents may come from teaching and research institutions in France or abroad, or from public or private research centers.
L'archive ouverte pluridisciplinaire HAL, est destinée au dépôt et à la diffusion de documents scientifiques de niveau recherche, publiés ou non, émanant des établissements d'enseignement et de recherche français ou étrangers, des laboratoires publics ou privés. 


\title{
A Bi-Criterion Approach for the Airlines Crew Rostering Problem
}

\author{
Walid El Moudani ${ }^{1,2}$, Carlos Alberto Nunes Cosenza ${ }^{3}$, Marc de Coligny ${ }^{4}$, \\ and Félix Mora-Camino ${ }^{1,2}$ \\ ${ }^{1}$ LAAS du CNRS, 7 Avenue du Colonel Roche, 31077 Toulouse, France. \\ \{wmoudani,mora\}@laas.fr \\ http://www.laas.fr \\ ${ }^{2}$ Air Transportation Department, ENAC/DGAC, 7 Avenue Edouard Belin, \\ 31055 Toulouse, France. \\ \{walid.elmoudani, felix.mora\}@enac.fr \\ http $/ /$ www.enac.fr \\ ${ }^{3}$ APIT, COPPE/UFRJ Centro de tecnologia, Rio de Janeiro, Brazil. \\ Cosenza@pep.ufrj.br \\ ${ }^{4}$ MIRA, Université de Toulouse II, France. \\ coligny@univ-tlse2.fr
}

\begin{abstract}
In this communication a bi-criterion approach for the nominal Airlines Crew Rostering Problem is developed. The nominal Crew Rostering Problem considers the assignment of the crew staff to a set of pairings covering all the scheduled flights so that operations costs are minimized while its solution must meet hard constraints resulting from the safety regulations of Civil Aviation as well as from the airlines internal agreements. Another goal is of the highest interest for airlines: since the overall satisfaction of the crew staff may have important consequences on the quality and on the economic return of the operations.

In this communication, a new mathematical formulation of the crew scheduling problem which takes into account the satisfaction of the crew members is proposed. A heuristic approach, combined with a genetic algorithms technique, is adopted to produce reduced cost solutions associated to acceptable satisfaction levels for the crew staff. The application of the proposed approach to a medium size Airline Crew Rostering Problem is evaluated.
\end{abstract}

Keywords. Multi-Criterion Optimization, Heuristics, Genetic Algorithms, Airlines Operations, Crew Scheduling, Crew Rostering.

\section{Introduction}

For more than three decades now the Airlines Crew Scheduling Problem (ACSP) has retained the attention of the Management and Operations Research community since crew costs in air transportation are extremely high, amounting $15-20 \%$ of total airlines operations costs. Therefore, airlines consider that the efficient management of their crew staff is a question of the highest economic relevance. Unfortunately, the exact 
numerical solution of the associated large scale combinatorial optimization problem is very difficult to obtain. Early rules of thumb [13] have been quickly overrun by the size of the practical problems encountered (hundreds or thousands of crew members to be assigned to at least as many pairings) and by the complexity of the set of constraints to be satisfied, leading very often to poor performance solutions. More recently, with the enhancement of computer performances, optimization approaches have been proposed to solve this problem : mathematical programming methods (large scale linear programming and integer programming techniques) (see [3], [4], [9], [10]), artificial intelligence methods (logical programming, simulated annealing, neural networks, fuzzy logic and genetic algorithms) as well as heuristic approaches and their respective combinations [11], [12], [15], [20]. Many studies refer to the nominal ACSP which is a static decision problem, based on a monthly table of flights, and devoted exclusively to the minimization of airlines operations costs. This problem is in general split in two sub problems: a crew pairing problem where the set of pairings covering the programmed flights is defined and a crew rostering problem where the retained pairings are nominally assigned to the crew staff.

In this paper, after discussing the airlines crew scheduling problem, the nominal Airline Crew Rostering Problem (ACRP) is introduced as a bi-criterion decision problem where the main decision criterion is the crew operations cost of the airline and the secondary decision criterion is relative to the crew staff overall degree of satisfaction. The solution approach proposed here is composed of two steps: in the first one a heuristic approach is designed to get a first set of high satisfaction assignment solutions and in the second one, an optimization process, based on genetic algorithms (GA) is developed. The application of this solution approach to a medium size ACRP is displayed.

\section{The Airline Crew Scheduling Problem}

The Airline Crew Scheduling Problem (ACSP) is treated in general once the schedule of the flights has been established for the next month and once the available fleet has been assigned to the scheduled flights. Two classes of constraints are considered in order to produce the "line of work" for the crew staff over the planning period : hard constraints whose violation impair the security of the flight (crew qualifications, national regulations concerning duration of work and rest times, medical clearances, training and license renewal requirements) and soft constraints (internal company rules, agreements with unions regarding the crew's working and remuneration conditions, office duties, holidays and declared assignment preferences by the crew staff) which are relevant to build the crew schedule but whose relaxation may lead to lower cost solutions. While some of these soft constraints are common to most airlines, others are only relevant for some classes of airlines and some few are specific to a given airline. The primary objective sought by airlines at this level of decision making is to minimize the crew related operations costs, so in most research studies, the ACSP has been formulated as a mono-criterion minimization problem. 
A sub optimal but widely accepted approach to tackle more efficiently the ACSP, which is of the NP-hard computational complexity class [8], consists in decomposing it in two sub-problems of lower difficulty. The first sub-problem, the Airline Crew Pairing Problem (ACPP), involves the construction of an efficient set of pairings (a pairing is a sequence of flights which starts and ends at the same airline base while meeting all relevant legal regulations) which covers the whole programmed flights. The second sub-problem, the Airlines Crew Rostering Problem (ACRP), considers the nominal assignment of the airline crew to the generated set of pairings over the planned period so that an effective "line of work" is obtained for each staff member (Fig. 1).

To get in a simple way a solution to the ACRP, most North American airlines have adopted in a first place some heuristics such as bidding processes where the crew staff is arranged by decreasing seniority and each crew member builds at his turn his own monthly "line of work" from the remaining pairings. This greedy heuristic approach generates too often uneven workloads and so induce repeated dissatisfaction among the crew staff. More recently, global approaches, based on Mathematical Programming techniques, have been proposed to tackle the ACRP [9].

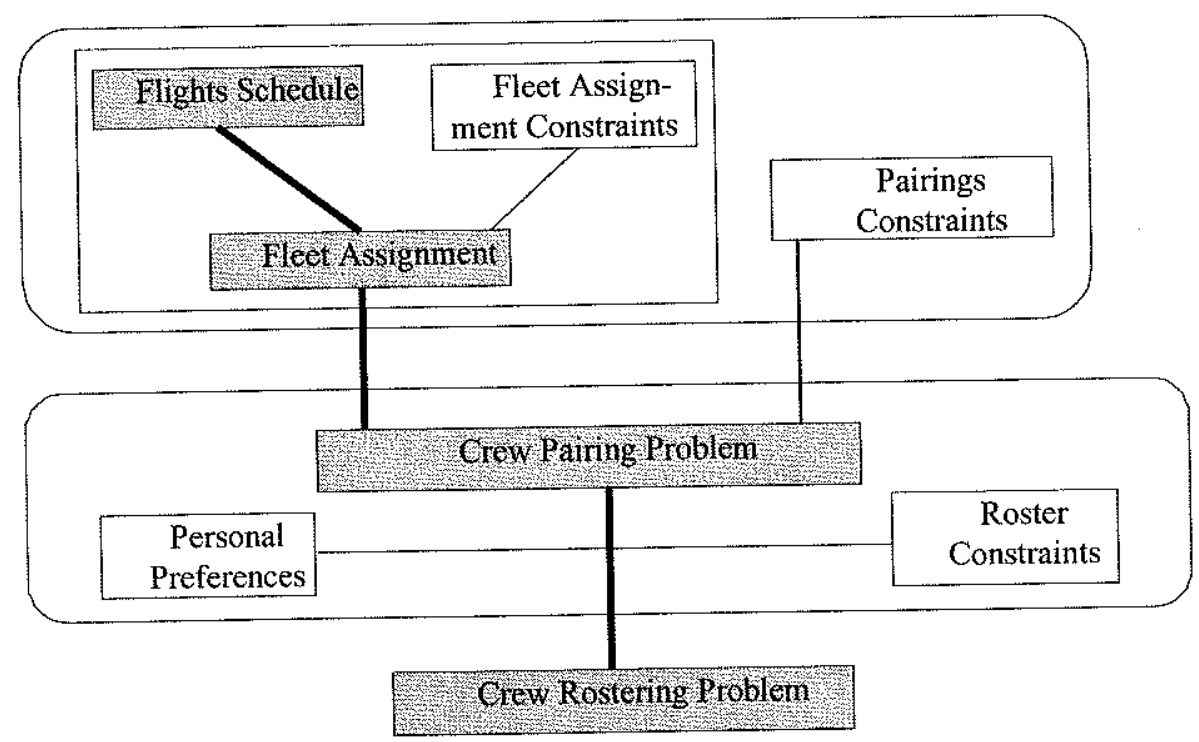

Fig. 1. The Airline Crew Scheduling Problem

\section{A Mathematical Programming Approach of the Nominal ACRP}

In this section a standard formulation of the ACRP is analyzed and the direct introduction of a crew satisfaction index is considered. 


\subsection{Analysis and Formulation of the Nominal ACRP}

The nominal Airline Crew Rostering Problem has been formulated as a zero-one integer mathematical programming problem [1], [13] where the crew operations cost is the criterion to be minimized under a finite set of hard constraints:

$$
\text { Minimize } \sum_{j \in J \in A_{j}} \sum_{i j} x_{i j}
$$

Subject to

$$
\begin{gathered}
\sum_{i \in A_{j}} x_{i j} \geq 1, \quad \forall j \in J \\
\left(x_{i j_{1}}+x_{i j_{2}}\right) \leq 1 \quad \forall j_{1} \in J, j_{2} \in O_{j_{1}}, i \in I \\
\sum_{j=1}^{m} d_{j} x_{i j} \leq L H \quad \forall i \in I \\
\sum_{j=1}^{m} x_{i j} \leq R_{\max }^{i} \quad \forall i \in I \\
x_{i j} \in\{0,1\} \quad \forall i \in I, \forall j \in J
\end{gathered}
$$

where $c_{i j}$ is the cost resulting from the assignment of pairing " $j$ " to pilot " $i$ ", $I$ is the set of the $n$ pilots, $J$ is the set of the " $m$ " pairings to be covered during the planning period, $A_{j}$ is the set of the pilots able to fly pairing " $j$ ", $O_{j 1}$ is the set of the pairings overlapping with pairing " $j_{1} ", d_{j}$ is the amount of flying hours associated to pairing " $j ", x_{i j}$ are binary variables such that $x_{i j}=1$ if pairing " $j$ " is assigned to pilot " $i$ ", $x_{i j}=0$ otherwise.

The first set of constraints ensures that to each pairing is assigned a unique crew, the inequality sign allowing crew deadheading (a transfer of crew members out of duty to another base in order to carry out a planned flight). The second set of constraints ensures that the same crew is not assigned to two overlapping pairings. The third set of constraints ensures that the number of hours flown by a pilot during the rostering period (a month in general) does not exceed an upper limit $L H$ and in the fourth set of constraints, $R_{\max }^{i}$ is the maximum number of pairings that can be assigned to crew member "i" over a rostering period. 
Some important comments can be done:

The cost resulting from the assignment of a pairing to a given crew member cannot be determined with precision before the whole workload of this crew member has been defined. In many airlines, crew payment is computed in a complex way from many parameters such as total amount of flown hours, standby duties and overtime. So, it appears that the above formulation can result in a poor approximation of the real assignment problem.

Other relevant constraints can be added as soft constraints to the mathematical formulation of the Airline Crew Rostering Problem. For instance, it has appeared important to many airlines to integrate in the ACRP the preferences of the crew staff. Effectively, in most airlines the crew staff can declare their preferences to carry out some pairings over the nominal rostering period, even if the final assignment decision is left to the airline operations management. Then, in addition to the minimum cost objective, it seems interesting to introduce a crew overall satisfaction objective either as a sub criterion or as a soft constraint.

Exact solutions for the resulting large scale combinatorial optimization problems are not available in an acceptable computing time and in general, airlines have produced their crew schedules using simplistic heuristics. However during the last decade, a number of major airlines have developed optimization-based techniques to solve the ACRP: improved heuristics based on column generation techniques and exact solution approaches based on constraints logic programming [8], [20].

\subsection{Introduction of the Crew Satisfaction Level}

For an airline, it is not easy to adopt a global index representative of the overall crew satisfaction level, the latter being composed of a large number of individual evaluations over different elements such as total flown time, total standby time and satisfaction of pairing preferences over the current and the last planning periods. A standby duty occurs when a pilot has to remain ready for the possible replacement of other unavailable crew members. The number of standby duties assigned to a given crew member over a planning period should remain small since this situation is not attractive, its hourly rate much lower than for the fights.

However, adopting a simplified approach, if it is possible to represent by a real number $S_{i j}$ the increment of satisfaction of crew member " $i$ " when he is assigned his requested pairing " $j$ " and by a real number $\overline{S_{i j}}$ the increment of satisfaction of crew member " $i$ " when he is not assigned pairing " $j$ " which he wants to avoid. Then the current degree of satisfaction of crew member " $i$ " can be given by:

$$
\operatorname{DOS}_{i}=\sum_{j \in J}\left(S_{i j} x_{i j}+\overline{S_{i j}}\left(1-x_{i j}\right)\right) \quad \forall i \in I
$$

Then it could be possible to introduce new restrictions to the nominal formulation of the ACRP, such as: 


$$
\operatorname{DOS}_{i}\left[x_{i j}, j \in J\right] \geq \operatorname{DOS}_{i}^{\min } \quad \forall i \in I
$$

to ensure a minimum degree of satisfaction for each crew member, and such as:

$$
\pi_{i k} * D_{D S}\left[x_{i j}, j \in J\right] \geq \operatorname{DOS}_{k}\left[x_{k j}, j \in J\right] \quad \forall i, k \in I, i \neq k
$$

where $\pi_{i k}$ is a relative seniority index, to ensure equity, tempered by seniority, between the different crew members.

This approach, although straightforward, increases notably the difficulty of the ACRP by introducing a large number of constraints in its formulation and needs the definition of many satisfaction-related parameters.

A first bi-criterion solution approach can adopt the following steps :

1) Solve by an approximate method the ACRP with restrictions (1) to (5), let $C_{\min }$ be the resulting minimum operations cost.

2) Choose a real number $\lambda$, superior to unity. Solve the following optimization problem:

$$
\begin{aligned}
\operatorname{Max} & \sum_{i \in I} w_{i}\left(\sum_{j \in J}\left(s_{i j} x_{i j}+\overline{S_{i j}}\left(1-x_{i j}\right)\right)\right) \\
\text { subject to constraints (1) to (5) and } & \sum_{j \in J \in A_{j}} \sum_{i=1 j} c_{i j} \leq \lambda C_{\min }
\end{aligned}
$$

where the $w_{i}$ are positive normalized seniority weights.

3) If this last constraint is active at solution, evaluate accurately the degree of satisfaction of each crew member and if too many crew members present a non adequate degree of satisfaction, increase parameter $\lambda$ and comeback to step 3 , otherwise, stop.

The main difficulties present in this first bi-criterion approach are related with the quantification of the satisfaction degrees (values of the $S_{i j}$ and $\overline{S_{i j}}$, analytical expression for the $D O S_{i}$ ) and the choice of the seniority weights which can hardly be non suggestive. 


\section{A New Solution Approach for the Nominal Airline Crew Rostering Problem}

It has been observed in section 3 that the cost structure of the ACRP is not separable with respect to pairings since to estimate accurately the operations cost associated with a given crew, his entire "line of work" for the next planning period must be known. Quite the same can be said about the crew individual and global satisfaction levels over a planning period. At this point, Genetic Algorithms techniques [15], [16], [17], [18] which manipulate complete solutions, appear interesting. [19] have used this property of AG to propose a new solution approach for the ACPP. Recall that the solution search process of $\mathrm{GA}$ is based on the stochastic improvement of a set of initial solutions ("the initial population") through the use of operators recalling the selection and evolution processes of natural species.

Here the ACRP is considered to present a main criterion, the airline operations cost, and a secondary criterion, the overall satisfaction degree of the staff. So, in order to generate a representative set of near Pareto solutions, it is proposed to start from a population of solutions obtained from a heuristic whose aim is to maximize the overall degree of satisfaction regardless of the operations cost, then, genetic techniques are used to generate new solutions sets with reduced operations cost over a sequence of «generations ». During this process, the levels of the degrees of crew satisfaction of the solutions composing the current generation suffer some abatement. The three mains stages of the proposed approach are showed in (Fig. 2).

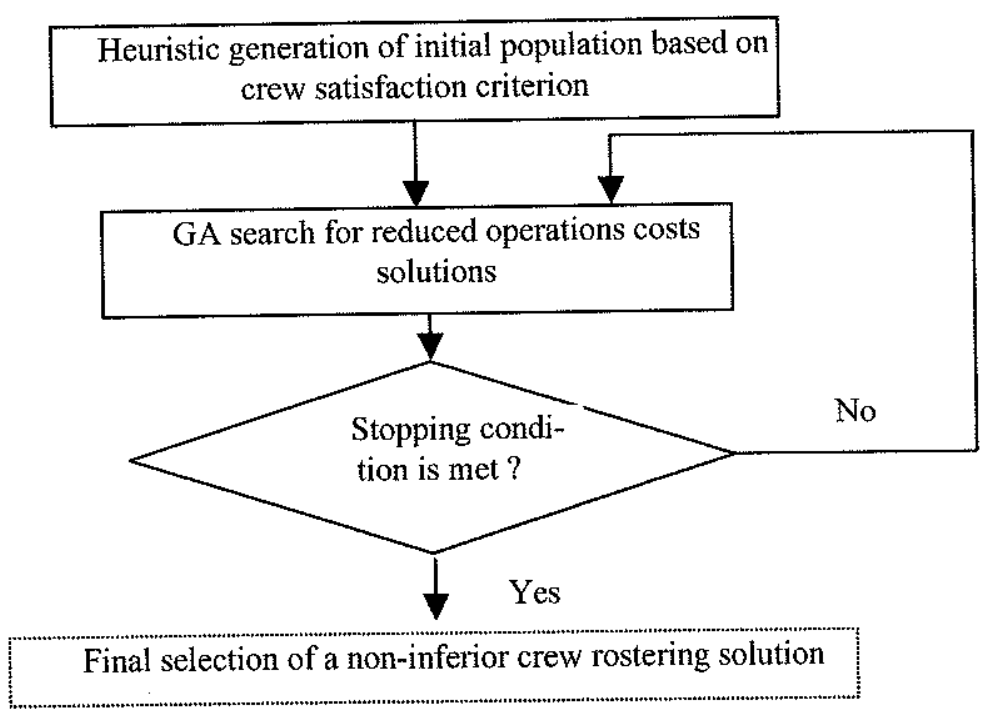

Fig. 2. The proposed GA-based solution approach 


\subsection{A Greedy Heuristic Approach to Build the Initial Population}

Once a representative index of the satisfaction of each crew member has been made available [22] for an approach based on fuzzy set theory), the initial solutions set can be built from a unique greedy heuristic technique (the Crew Satisfaction HeuristicCSH) applied to different arrangements of the set of crews. Note that the satisfaction of the crew builds up also from past assignments to different activities. It is supposed here that at the beginning of a new planning period each crew is characterized by a degree of satisfaction belonging to a qualitative scale such as $\{$ VeryLow, Low, Medium, Fair,High,VeryHigh\}. With the proposed greedy heuristic, the preferences of crew members of lowest satisfaction degree are considered first. This heuristic technique is divided in three stages. In the first stage, the crew staff of lowest satisfaction are assigned, when possible, to their requested pairings. In the second stage, the remaining rejected pairings are assigned to the crew staff who do not bother with them. However, if all remaining crew members wish to avoid some pairings, then the crew members with the highest satisfaction level will have to cope with them. Finally, the rest of the remaining pairings is assigned to the crew members in order to complete in a balanced way their "line of work".

To build the initial population this process must be repeated until the desired size of the population has been reached. Since in each satisfaction class ' $i$ ' of cardinal " $n_{i}$ ", there are $n_{i}$ ! different arrangements and since " $n_{i}$ " can be high, the number of different arrangements can be an extremely high number $\left(\prod_{i=1}^{5} n_{l} !\right)$. So, if sometimes, the heuristic assignment process produces identical assignment solutions, it is easy to find by random another arrangement in each satisfaction class, so that a new initial assignment solution is obtained.

Since the set of pairings can be structured as a directed graph when temporal precedence, reachability and overlapping constraints are considered (see table 1 where $X^{-}$and $X^{+}$represent the starting and the completion times of pairing $X$ ), each element of the initial population can be represented by a set of " $n$ " independent paths in the corresponding pairings precedence graph (PPG).

Table 1. Precedence and overlapping relations

\begin{tabular}{|c|c|}
\hline Relation & Temporal conditions \\
\hline$X$ before $Y$ & $X^{-} \prec X^{+} \prec Y^{-} \prec Y^{+}$ \\
\hline$X$ meets $Y$ & $X^{-} \prec X^{+}=Y^{-} \prec Y^{+}$ \\
\hline$X$ overlaps $Y$ & $X^{-} \prec Y^{-} \prec X^{+} \prec Y^{+}$ \\
\hline$Y$ finishes $X$ & $X^{-} \prec Y^{-} \prec X^{+}=Y^{+}$ \\
\hline$Y$ during $X$ & $X^{-} \prec Y^{-} \prec Y^{+} \prec X^{+}$ \\
\hline$X$ starts $Y$ & $X^{-}=Y^{-} \prec X^{+} \prec Y^{+}$ \\
\hline$X$ equals $Y$ & $X^{-}=Y^{-} \prec X^{+}=Y^{+}$ \\
\hline
\end{tabular}




\subsection{A New GA Solution Strategy for the Nominal Airline Crew Rostering Problem using GA}

Genetics Algorithms process sets of feasible solutions, called "populations" where each element of these populations is similar to a chromosome composed of genes, each gene corresponding to a particular parameter of the problem. The chromosomes can be represented using either a binary or a non-binary codification.

For the problem tackled here, a non-binary codification has been adopted for the representation of the solutions composing a given population. The $i^{\text {th }}$ component of a « chromosome » indicates which crew member is assigned to the $\mathrm{i}^{\text {th }}$ pairing (Fig. 3). The size of a population considered in the case studied here is equal to 30 chromosomes.

1
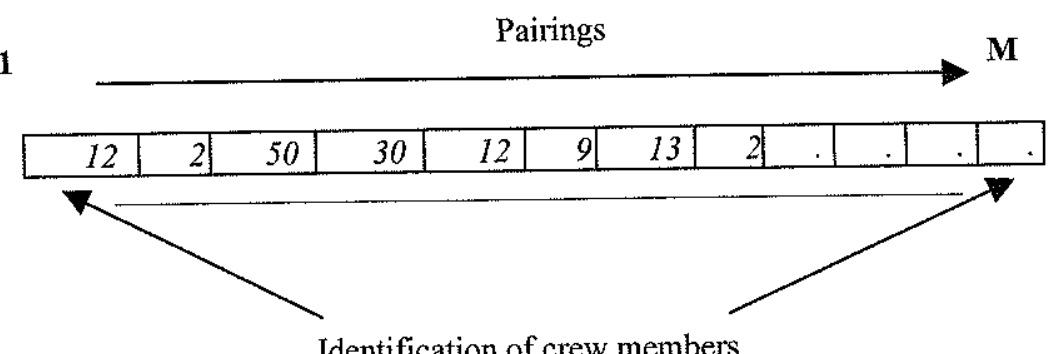

Identification of crew members

Fig. 3. The adopted coding for an ACRP solution

This codification has been chosen in order to minimize the memory requirements to codify a whole population, however, it is straightforward to associate by inspection, to each of these chromosomes, a set of " $n$ " independent paths in the PPG (Fig. 4).

An operator select the chromosomes which become parents according to their evaluation values. A roulette wheel method picks two chromosomes to which classical GA operators like crossover, inversion and mutation are applied according to a chosen probability.

Classical genetics operators (crossover, mutation and inversion) have been adapted to the context of the present assignment problem to produce in a progressive way improved new generations. Each elementary genetic operation processes indirectly the pairing assignments of two different pilots. When a noticeable improvement of the operations cost is obtained, the local modification is retained, but when local solution costs are equivalent, the respective degrees of satisfaction of the two pilots are taken into consideration to make the choice.

The stopping rule adopted here is based not only, as usual with GA, on the allowed maximum number of non-improving successive generations, but also on the proportion in the current population of the cost effective solutions for which too many individual degrees of satisfaction are insufficient ("Low" or "Very Low"), since these cost effective solutions will represent a high risk for the airline. 


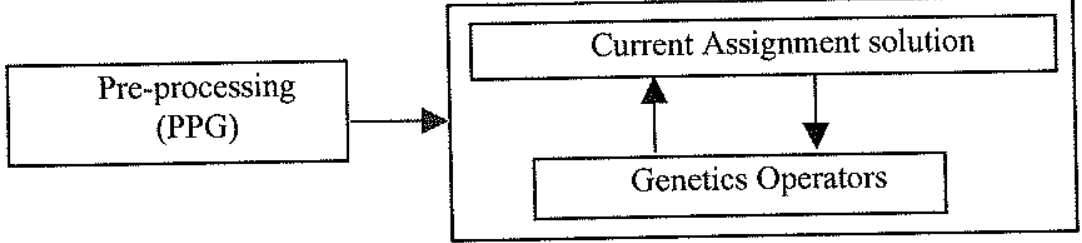

Fig. 4. Feasibility of the assignment solutions

\subsection{Problem-Specific Mutation and Inversion Operators}

In order to speed up the discovery of the most promising assignment solutions, a local heuristic technique has been introduced to restrict the search space of the mutation and the inversion processes. It consists in dividing the crew members in two sets, $W L_{L}$ and $W L_{H}$, corresponding to workloads less and higher than the current average workload which is computed for the whole staff.

With respect to the mutation operator, a position " $k$ " is chosen at random in the chromosome, the crew member " $x$ " found in the position " $k$ " will be replaced by another crew member " $y$ " chosen randomly in set $W L_{L}$ and for which this new task is feasible (the precedence constraints in the PPG must be checked for that). The idea is here to contribute actively to a more balanced workload over the whole staff. Then the solution under treatment by the mutation operator suffers the following modification:

$$
\begin{aligned}
& \forall x \in W L_{L} \cup W L_{H} \text { then } \exists y \in W L_{L} / S O L[k]=y \text { with } \\
& \quad W L_{x}=W L_{x}-d r^{k}, W L_{y}=W L_{y}+d r^{k} \quad \forall k \in\{1, \cdots N\}, x \neq y
\end{aligned}
$$

where $S O L[k]=y$ means that the assignment solution array $S O L$ contains the crew member " $y$ " in the position " $k$ " and $d r k$ is the duration of the pairing " $k$ ", i.e. the pairing " $k$ " of duration $d r$ is non assigned to crew member " $y$ ".

In the case of the inversion operator, a position " $i$ " corresponding to pairing " $i$ " is chosen randomly, if it is assigned to a crew member " $x$ " belonging to $W L_{H}$ then a crew " $y$ " belonging to $W L_{L}$ is chosen at random, while a pairing " $j$ " performed initially by crew member " $y$ " is selected randomly. If its duration is less than the duration of pairing " $i$ " and the inversion is feasible, then a new solution is produced where:

$$
S O L[i]=y \text { and } S O L[j]=x \quad \forall i, j \in\{1, \cdots N\} i \neq j, x \neq y
$$


In the case where position " $i$ " corresponds to a crew belonging to $W L_{L}$, the above process is inverted.

The final assignment solutions are arranged in accordance with the criterion cost and, when equality of costs, with respect to an overall satisfaction degree criterion.

\section{Case study}

This solution approach has been already applied to a medium size problem where 75 crew members must be assigned to 275 pairings corresponding to a total amount of 4250 flight hours. Some learning can be obtained from this first application with respect to the computer effectiveness of the proposed approach and with respect to the quality of the solution set obtained.

\subsection{Computer Effectiveness Results}

When applying the first step of the proposed approach, the satisfaction-based computation of the initial population through the CSH greedy heuristic is immediate and requires a relatively short computing effort. In relation to the subsequent genetic algorithm, it appears that the different genetic operators do not present equivalent performances: the crossover operator, which is quite computer time consuming, does not contribute too much to produce new promising assignment solutions since the set of constraints to be checked is very large, while the mutation and inversion operators appear to be more efficient to generate new assignment solutions with relatively moderate computing times. The genetic operators are applied according to the probabilities $p_{c}=0.20, p_{m}=0.40$ and $p_{i}=0.40$.

To compare the efficiency of the different genetic operators combinations over the final cost effective solutions, some parameters have been introduced: the workload deviation index $\sigma_{W L}$ and the proportion of non satisfied crews $\tau_{N S}$. Here $\sigma_{W L}$ is given by:

$$
\sigma_{W L}=\sqrt{\sum_{i=1}^{n}\left(W L_{i}-\overline{W L}\right)^{2} / n}
$$

where $W L_{i}$ is the workload assigned to crew member " $i$ ", $\overline{W L}$ is the average workload for the rostering period and " $n$ " is again the size of the staff. The comparative results are displayed in Table 2. 
Table 2. Effectiveness of CSH and genetic processes for the ACRP

\begin{tabular}{|c|c|c|c|}
\hline & $\begin{array}{c}\text { Minimum } \\
\text { Cost } \\
\text { Solution }\end{array}$ & $\begin{array}{c}\sigma_{W L} \\
\text { Cost Disper- } \\
\text { sion } \\
\end{array}$ & $\begin{array}{l}\text { Proportion of } \\
\text { non satisfied } \\
\text { crews }\left(\tau_{N S}\right)\end{array}$ \\
\hline CSH Heuristic & $4312: 30$ & $4: 50$ & 0.13 \\
\hline Crossover alone & $4304: 15$ & $4: 35$ & 0.25 \\
\hline Mutation alone & $4290: 30$ & $3: 30$ & 0.49 \\
\hline Inversion alone & $4291: 00$ & $3: 35$ & 0.42 \\
\hline Crossover + Mutation & $4278: 30$ & $3: 20$ & 0.48 \\
\hline
\end{tabular}

\subsection{Analysis of the Assignment Solutions}

In figure 5, the total cost and the proportion of non satisfied crew distributions of the final population are displayed to provide an idea of the non inferior set of solutions to the considered problem. In figures $6 . a$ and $6 . \mathrm{b}$, the individual workloads and satisfaction degrees corresponding to its extreme solutions are displayed: $S_{L}$ and $S_{H}$. In figures 7.a and 7.b, the individual workload distribution generated after using CSH and GA are displayed.

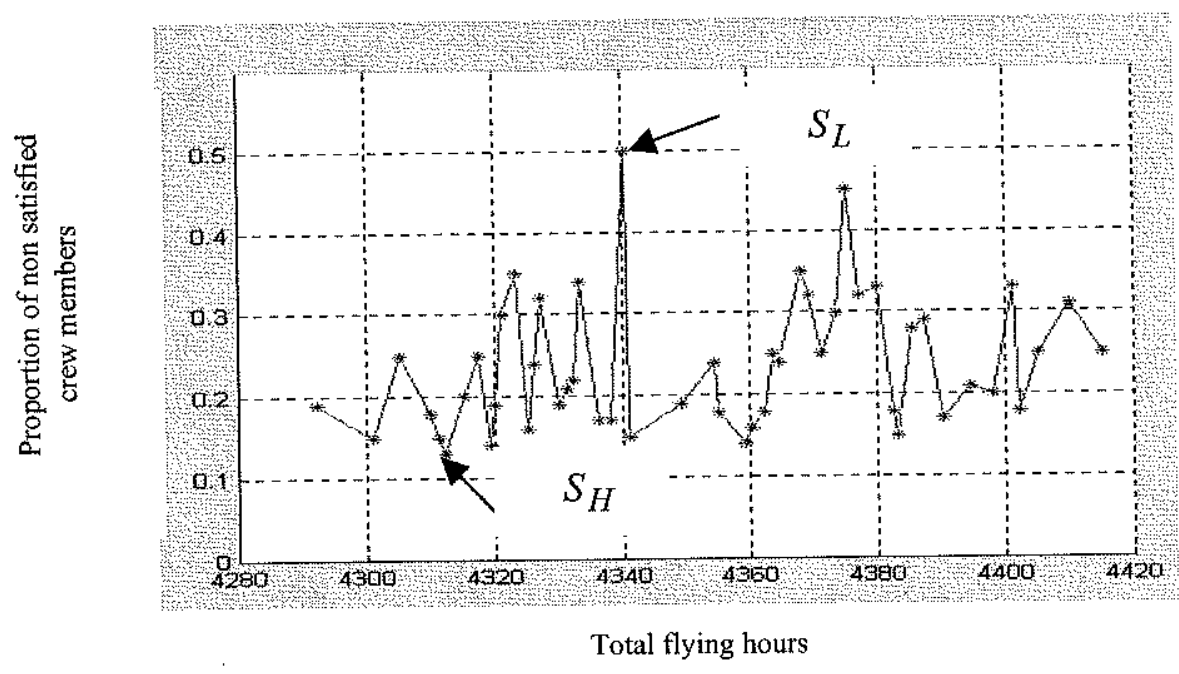

Fig. 5. Final solution set 


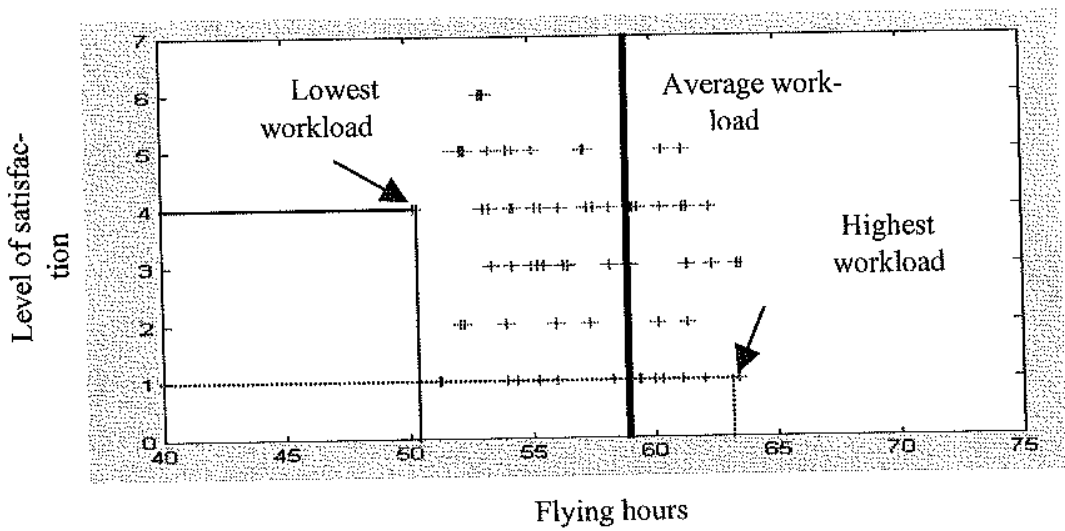

Fig. 6.a. Solution corresponding to $S_{H}$

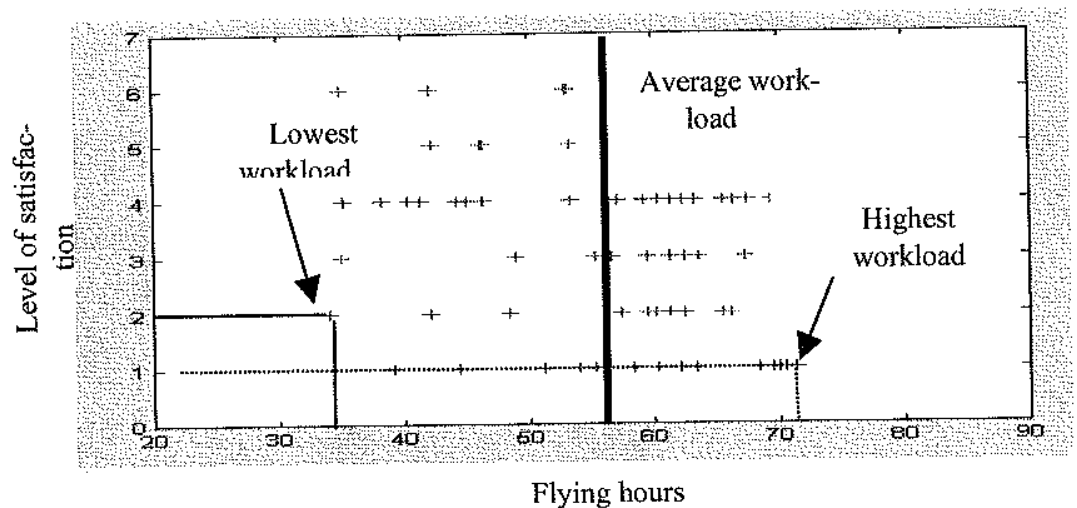

Fig. 6.b. Solution corresponding to $S_{L}$

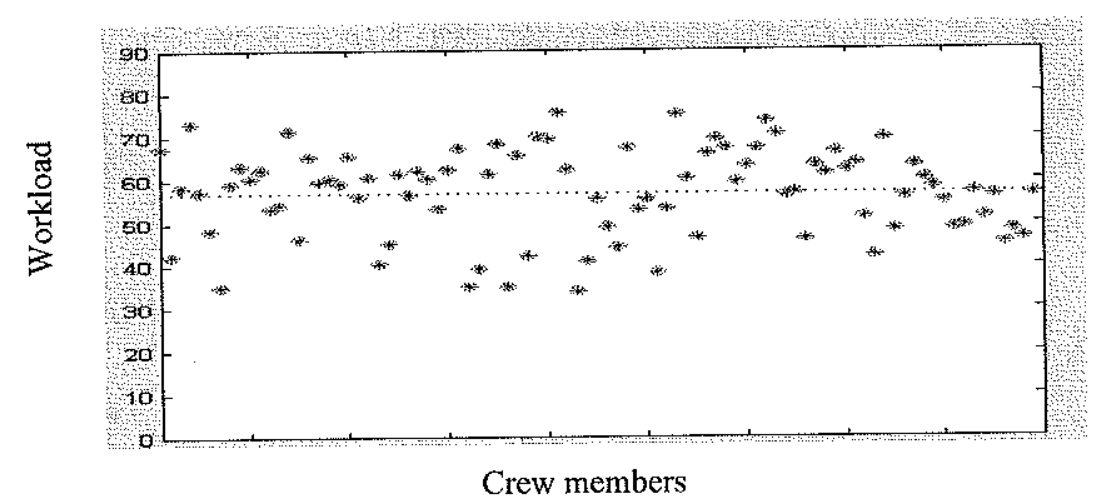

Fig. 7.a. Example of workload distribution corresponding to the greedy heuristic 


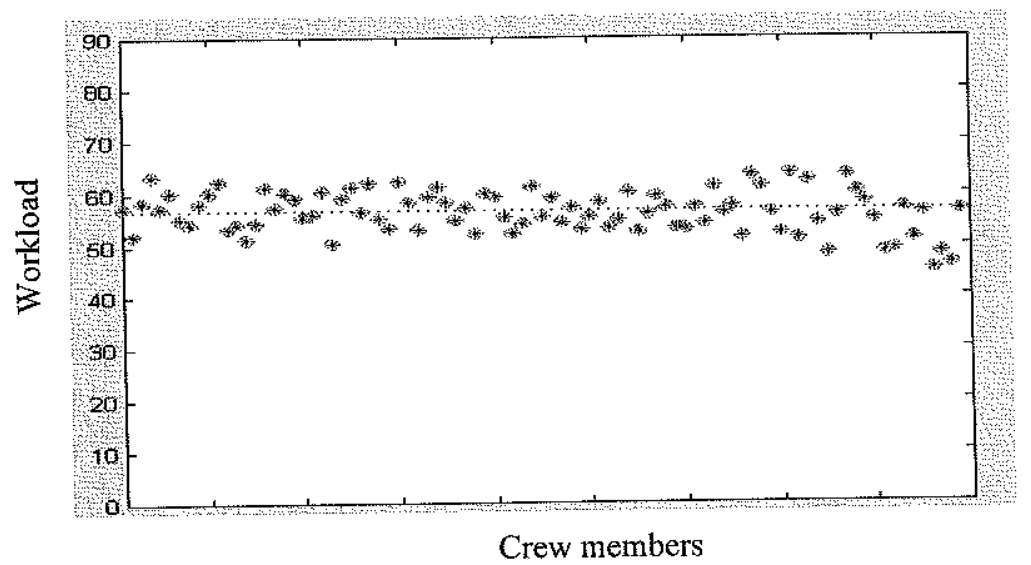

Fig. 7.b. Example of final workload distribution with $\mathrm{GA}\left(\mathrm{S}_{\mathrm{L}}\right.$ solution)

\section{Conclusion}

In this communication, one of the main operations decision problem faced by airlines has been dealt with using Mathematical Programming and Computational Intelligence methods. The proposed approach does not produce an exact solution in pure mathematical terms but appears to be quite adapted to give a real support to decision making, by providing, through a comprehensive process, an improved approximation of the set of non inferior solutions attached to this bi-criterion decision problem.

\section{References}

1. Arabeyre, J.P., Fearnley, J., Steiger F.C.: The Airline Crew Scheduling Problem: A Survey. Transportation Science, 1 (1969) 140-163.

2. Baker, E., Bodin, L., Fisher, M.: The Development and Implementation of a Heuristic Set Covering Based System for Air Crew Scheduling. Management Science and Statistics Working Paper 80-015, College of Business and Management, University of Maryland (1980).

3. Ball, M. and Roberts, A.: A Graph Partitionning Approach to Airline Crew Scheduling. Transportation Science, 19/2 (1985) 107-126.

4. Desaulniers, G., Desrosiers, J., et al.: Crew pairing at Air France. European Journal of Operational Research, (1997) 245-259.

5. EL Moudani, W. and Mora-Camino, F.: Airlines Fleet Operations Management : An Integrated Solution Approach. XV IEE on CAD/CAM, Aguas de Lindoia, SP, Brazil (1999). 
6. EL Moudani, W. and Mora-Camino, F.: A Dynamic Approach for Aircraft Assignment and Maintenance Scheduling by Airlines. Journal of Air Transport Management, 6/4, (2000) 233-237.

7. EL Moudani, W. and Mora-Camino, F.: A Fuzzy Solution Approach For The Roster Planning Problem. The $9^{\text {th }}$ IEEE International Conference on Fuzzy Systems, San Antonio, Texas, USA (2000).

8. Mora-Camino, F.: Modélisation, Optimisation, complexité et Algorithmes. Lectures Notes, CNAM-Toulouse, France (1997).

9. Gamache, M., Soumis, F., Marquis, G., Desrosiers J.: A Column Generation Approach for Large Scale Aircrew Rostering Problems. Les Cahiers du GERAD, G94-20, Ecole des Hautes Etudes Commerciales, Montréal, Canada, H3T IV6 (1994).

10. Crainic, T.G. and Rousseau, J.M.: The Column Generation Principle and the Airline Crew Scheduling Problem. INFOR 25 (1987) 136-151.

11. Byrne, J.: A Preferential Bidding System for technical Aircrew (QANTAS AUSTRALIA). The $28^{\text {th }}$ AGIFORS Symposium, Massachusetts, US (1988).

12. Sarra, D.: The automatic assignment model (SATURN - ALITALIA). The $28^{\text {th }}$ AGIFORS Symposium, Massachusetts, US (1988).

13. Rubin, J.: A Technique for the Solution of Massive Set Covering Problems, with Application to Airline Crew Scheduling. Transportation Science, 7 (1973) 34-48.

14. Campello, R.E. and Maculan, N.: Algoritmos e heuristicas. Ed. Universidade federal Fluminense, Niteroi, Brazil (1994).

15. Teodorovic, P. and Lucic, P.: A fuzzy set theory approach to the aircrew rostering problem. Fuzzy Sets and Systems, 95 (1998) 261-271.

16. Holland, J.H.,; Adaptation in Natural and Artificial Systems. University of Michigan Press, Ann Arbor (1975).

17. Goldberg, D.E.: Genetic Algorithms in search, optimization and machine learning. Addison-Wesley, Reading (1989).

18. Bridges, C.L. and Goldberg, D.E.: An Analysis of Reproduction and Crossover in a Binary-Coded Genetic Algorithm. Proceedings of the second International Conference on Genetic Algorithm. ICGA (1987).

19. Bridges, C.L. and Goldberg, D.E.: An Analysis of Multipoint Crossover. Proceedings of the Foundation of Genetic Algorithms. FOGA (1991).

20. Beasly, J.E. and Chu, P.C.: A Genetic Algorithm for the Set Covering Problem. European Journal of Operational Research, 94 (1996) 392-404.

21. Guerinik, N. and Van Caneghem, M.: Solving Crew Scheduling Problem by Constraint Programming. Proceedings of the $1^{\text {st }}$ Conference of Principles and Practice of Constraint Programming, (1995) 481-498.

22. EL Moudani, W. and Mora-Camino, F.: Evaluation par la Logique Floue de la satisfaction du personnel navigant technique dans les compagnies aériennes. Rapport LAAS-CNRS, Toulouse, France, Déc. 2000. 